\title{
Effectiveness of a Rubber Band for Improving the Usability of a Smartphone
}

\author{
Noriyasu Hirokawa (hirokawa@waka.kindai.ac.jp) ${ }^{1}$, Yuki Sasaki (h13015sy@waka.kindai.ac.jp)1, \\ Yuki Tsumura (h13081ty@waka.kindai.ac.jp) ${ }^{1}$, Tsutomu Nishigaki (nisigaki@waka.kindai.ac.jp) ${ }^{1}$, \\ Mitsushi Ohmasa (ohmasa@waka.kindai.ac.jp) ${ }^{1}$ \\ ${ }^{1}$ Department of Human Factors Engineering and Environmental Design, Kindai University, Kinokawa, 649-6493
}

\begin{abstract}
The size of smartphones has been increasing and many users find them inconvenient to operate. When a user touches an icon that is located on the side away from their hand, at the bottom of the panel, or close to their hand, they may have to stretch, abduct, or bend their thumb, causing discomfort. The situation is extreme when the user inputs letters because the keys are small. By using an accessory such as a ring or hand strap, a user can hold a smartphone in a different posture compared to that used without an accessory so as to operate the smartphone more easily. This study investigates the effectiveness of a rubber-band accessory by measuring the IP-joint and CM-joint angles when a subject touches four keys located at the corners of a Japanese keypad.
\end{abstract}

Keywords: Usability, Smartphone, Accessory, Joint angle, Holding posture

\section{Introduction}

Many people use one hand to operate their smartphone; when they need to touch a key located at the side of the phone away from their hand, at the bottom of the panel, or very near to their hand, they have to stretch, abduct, or bend their thumb, respectively (Hirokawa et al., 2013, Hirokawa et al., 2015). As smartphones have increased in size to show more information, people have found them difficult to operate using their thumb while holding the phone. For example, a person with smaller hands cannot grab a larger-sized smartphone and must use their fingers, reducing stability and increasing the risk of dropping it; otherwise, they may have difficulty stretching their thumb to touch keys that are far, particularly when inputting letters because a user has to touch small keys exactly. Therefore, it is necessary to develop a method of holding a smartphone and operating it easily using one hand.

Recently, several smartphone accessories, such as rings and hand straps, have been developed to improve smartphone usability. Using such accessories, a person can hold a smartphone stably with their fingers even if it is too large for them to grab. The user can hold it so as to touch keys even if they are in the extreme position of inputting with one hand.

This study investigates the posture of the thumb when a smartphone user inputs Japanese letters with one hand and reveals the effectiveness of a rubber-band accessory.

\section{Input methods for Japanese letters}

\subsection{Japanese keypad}

To input Japanese letters on a smartphone, many people use a Japanese syllabary, a table of Japanese phonograms. In a Japanese syllabary, five vowel letters are aligned in the order of "A," "I," "U," "E," and "O" in "A" row. Consonant letters are aligned in this vowel order, "KA," "KI," "KU," "KE," and "KO" in "KA" row, "K"-sound letters, as an example. Figure 1(a) shows an example of a Japanese keypad based on a Japanese syllabary. In this keypad, each syllable with an "A"-sound is represented.

A user can input "A"-sound letters by touching the key, and can input other "I"-, "U"-, "E"-, and "O"'-sound letters by toggling or flicking the appropriate key. With a toggle-input system, every time the user touches the key, a candidate letter is changed to "I"-, "U"-, "E"-, and "O"-sound letters, and the user touches the key several times until the input letter emerges. By using the flick-input system, when the user touches a key, "I"-, "U"-, "E"-, and "O"-sound letters emerge around 
the key, as shown in Fig. 1(b), and the user flicks the finger to the direction in which the input letter is located.

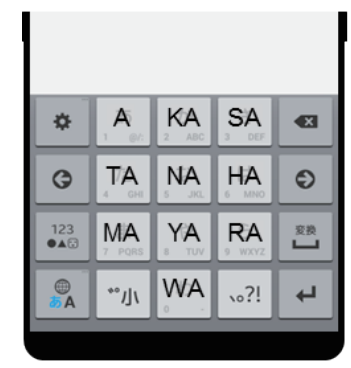

(a) Japanese keypad

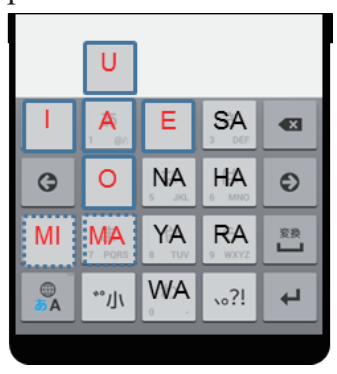

(b) Flick-input system
Figure 1. Inputting Japanese letters

\subsection{Thumb's posture when inputting a letter}

When a user holds a smartphone with their right hand and inputs "I"-sound letters with their thumb using a flick-input system, they must stretch their thumb and move it away from their holding hand. This is especially true if the smartphone in question is large. Comparing the postures of the thumb when inputting an "I"-sound letter on narrow and wide smartphones, as shown in Figs. 2(a) and (b), it can be seen the user has to move their thumb more on a wide smartphone, reducing usability.

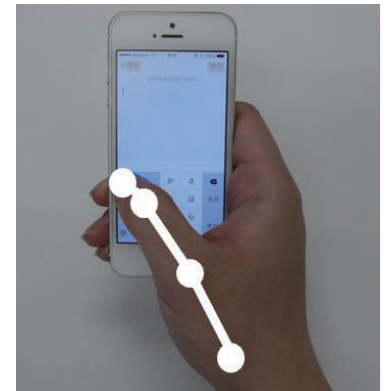

(a) Narrow smartphone

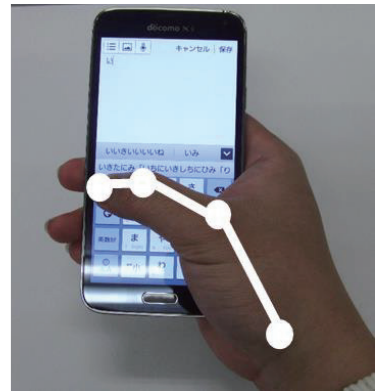

(b) Wide smartphone
Figure 2. Posture of the thumb in inputting a letter

\subsection{Holding posture}

Figure 3 shows the hand posture in holding a smartphone without an accessory (Fig. 3(a)), with a ring-type accessory (Fig. 3(b)), and with a rubber-band accessory (Fig. 3(c)). In this study, the position of the tip of the thumb when a user holds a smartphone is called the neutral position and denoted by "NP." In Fig. 3(a), the hand grabs the smartphone and the NP is above the Japanese keypad, with the thumb located a little to the right-hand side. In Fig. 3(b), although NP is almost the same as that in Fig. 3(a), the thumb's posture is a little nearer to the center of the keypad area. In Fig. 3(c), the right hand does not

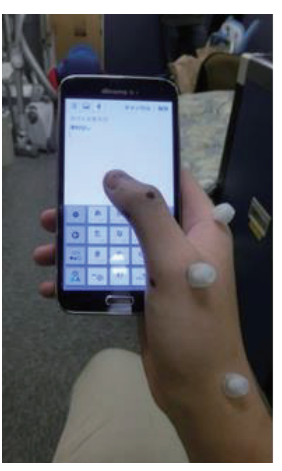

(a) without an accessory

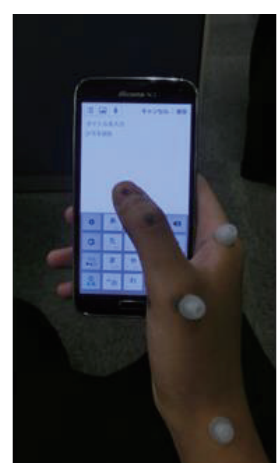

(b) with a ring-type accessory

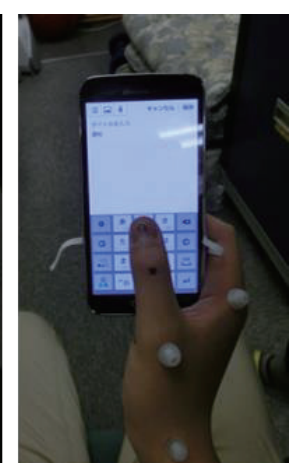
accessory (c) with a rubber-band

Figure 3. Holding posture of a smartphone

grab the smartphone and the NP is located within the Japanese keypad and the posture of the thumb is different from those of Figs. 3(a) and 3(b). The reason is that, when using a rubber-band accessory, the user does not have to grab the smartphone and can hold it so as to touch the keys easily.

\section{Experiment}

\subsection{Estimation of joint angles}

This study measures the angles of the IP- and CM-joints (Rohen, 2007, Netter, 2007) when a subject touches keys at the corners of the Japanese keypad and estimates the load in touching them. First, markers are attached at the IP- and CM-joints; then, the angles are measured using photographs taken from the perpendicular direction while the subject touches keys.

Figure 4 shows a photograph of the thumb while a subject touches a key. The photograph is taken from the direction perpendicular to the flat surface that includes the thumb's tip, IP-joint, and MP-joint, which are labeled $\mathrm{P}_{1}, \mathrm{P}_{2}$, and $\mathrm{P}_{3}$ in the figure, respectively. The IP-joint angle $\mathrm{P}_{1} \mathrm{P}_{2} \mathrm{P}_{3}$ is calculated by applying the law of cosines to the triangle $-\mathrm{P}_{1} \mathrm{P}_{2} \mathrm{P}_{3}$.

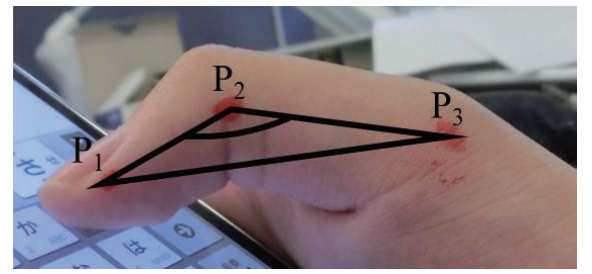

Figure 4. IP-joint angle while a subject touches a key 
Figures 5(a) and (b) show the thumb when it touches a key. Figure 5(a) is taken from the direction perpendicular to the flat surface that includes the MP-joints of the thumb and the index finger and the CM-joint of the hand, and Fig. 5 (b) is taken from a direction parallel to the panel. The MP-joints of the thumb and index finger are called $\mathrm{Q}_{1}$ and $\mathrm{Q}_{2}$, and the CM-joint is called $\mathrm{Q}_{3}$. The origin of the coordinate system is allocated at the top-left corner of the panel and the $x-, y$ - and z-axes are directed to the right, to the bottom, and above, respectively. (1) First, the reduction scales of the two photographs are calculated by the ratio between the lengths on the photograph and the corresponding true lengths. (2) Next, the $\mathrm{x}-$, y- and $\mathrm{z}$-coordinates at the markers on the photograph, $\mathrm{Q}_{1}\left(x_{1}, y_{1}\right.$, $\left.z_{1}\right), \mathrm{Q}_{2}\left(x_{2}, y_{2}, z_{2}\right)$, and $\mathrm{Q}_{3}\left(x_{3}, y_{3}, z_{3}\right)$, are measured. (3) Then, their true values, $\mathrm{Q}_{1}\left(X_{1}, Y_{1}, Z_{1}\right), \mathrm{Q}_{2}\left(X_{2}, Y_{2}, Z_{2}\right)$, and $\mathrm{Q}_{3}\left(X_{3}\right.$, $Y_{3}, Z_{3}$ ), are calculated using the reduction scales. (4) Finally, the angle $\mathrm{Q}_{2} \mathrm{Q}_{3} \mathrm{Q}_{1}$ at the $\mathrm{CM}$-joint is calculated by applying the law of cosines to the triangle- $\mathrm{Q}_{1} \mathrm{Q}_{2} \mathrm{Q}_{3}$.

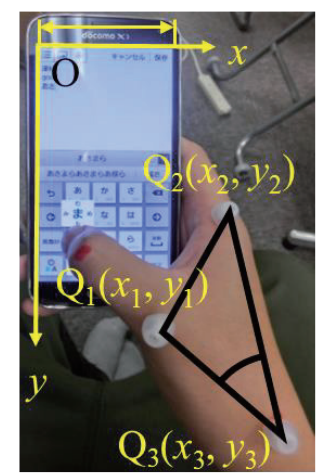

(a) top view

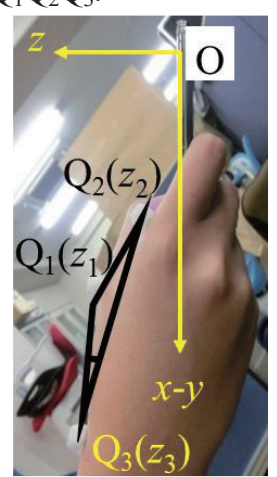

(b) side view
Figure 5. CM-joint angle as a subject touches a key

\subsection{Experimental conditions}

Two 21-year-old male students (subjects-A and -B) participated in our experiments. The Docomo Galaxy S5 produced by Samsung is used as the smartphone. Its body height, width and depth are about $142 \mathrm{~mm}, 73 \mathrm{~mm}$, and 8.3 $\mathrm{mm}$, respectively; its weight is about $147 \mathrm{~g}$. The height and width of the touch panel are $113 \mathrm{~mm}$ and $63 \mathrm{~mm}$, respectively, and the height and width of the Japanese keypad area, which is located in the lower position of the touch panel, are $49 \mathrm{~mm}$ and $63 \mathrm{~mm}$, respectively.

In the experiment, the subjects attach a rubber band to the rear surface of the smartphone where they like, as shown in Fig. 6(a), and twist it around their middle fingers, as shown in Fig. 6(b).

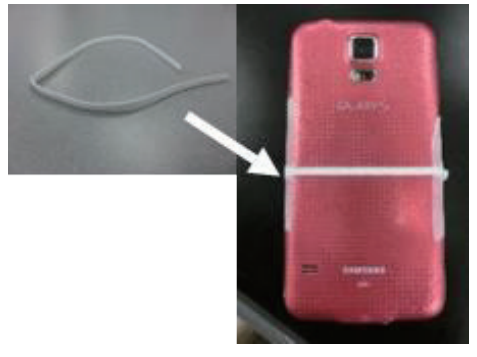

(a) Smartphone with a rubber band

(b) Holding posture

\section{Results}

This paper reports the results for subject-A of the restriction of the space. Figures 7 shows the IP-joint angles of subject-A in touching four keys: "A," "SA," "MA," and "RA," compared with those in neutral position. In the figures, smaller angles indicate that the subject bends the IP-joint more and needs a greater load. The dotted line on each finger indicates the IP-joint angle in the neutral position and the difference between the dotted line and the top of the bar indicates the bend angle of the IP-joint needed to touch the key. From Figs. 7(a) and 7(b), the subject has to bend the IP-joint more when he does not use an accessory or used a ring-type accessory. However, from Fig. 7(c), he does not have to bend the IP-joint when he uses a rubber-band accessory. Figure 8 shows the CM-joint angle of subject-A in the same style. In the figures, larger angles mean the subject abducts the CM-joint more and takes a greater load. From Fig. 8(a), the subject has to abduct the CM-joint when he does not use an accessory, but does not have to abduct it at all for either the ring-type or rubber-band accessories (Figs. 8(b) and 8(c)).

Figure 9 shows the relationship between the angles of the IP- and CM-joints when touching each of the four keys. The horizontal and vertical axes indicate the differences in the IP-joint and CM-joint angles from the case of the neutral position. Positive differences in these angles mean that the subject must bend or abduct more than he does at NP, respectively. In the figure, the blue markers (without an accessory) are spread widely in the upper-right area, indicating that the subject simultaneously bent the IP-joint and abducted the CM-joint. The gray markers (with a ring-type accessory) exist around the horizontal axis, which indicates that the subject bent the IP-joint but did not abduct the CM-joint. The orange 


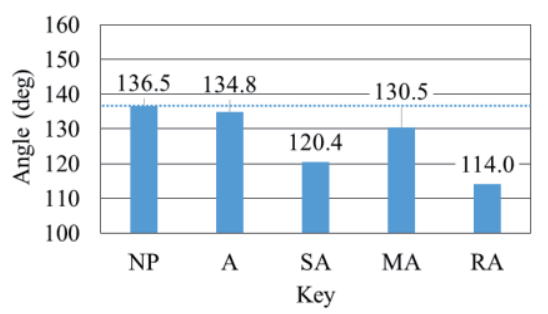

(a) without an accessory

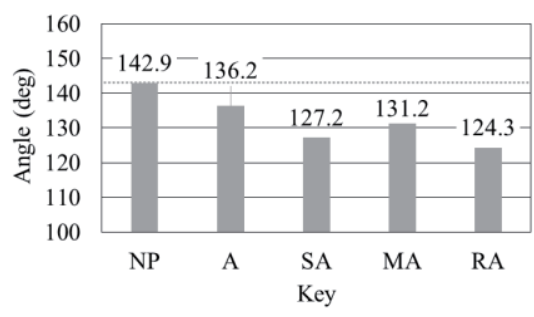

(b) with a ring-type accessory

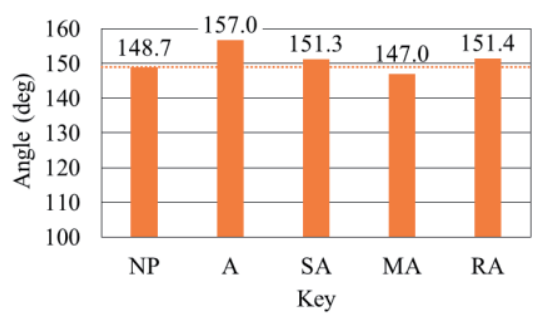

(c) with a rubber-band accessory

Figure 7. IP-joint angles when touching the four keys

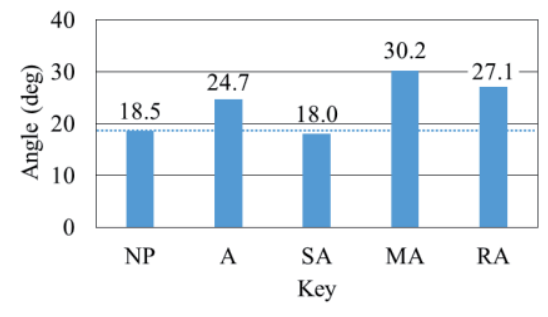

(a) without an accessory

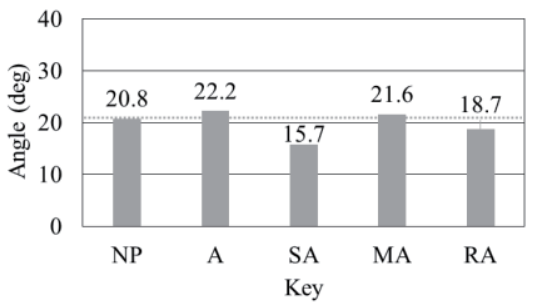

(b) with a ring-type accessory

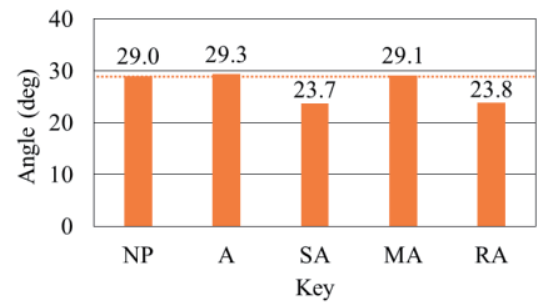

(c) with a rubber-band accessory

Figure 8. CM-joint angles when touching the four keys

markers (with a rubber-band accessory) gathered near the original point indicate that the subject did not bend the IP-joint or abduct the CM-joint.

By using a rubber band accessory, users can rotate the smartphone such that they can operate it easily without bending or abducting their thumb as shown in Fig. 10.

without accessory $\bigcirc$ with ring-type $\bigcirc$ with rubber-band

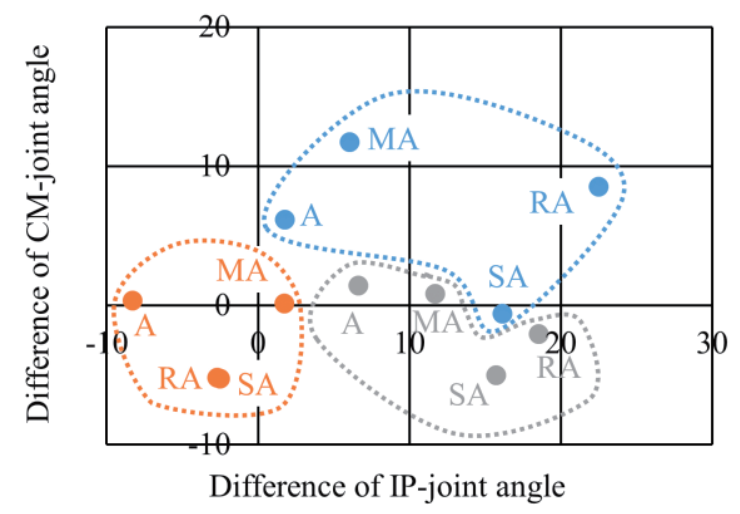

Figure 9. Angle distributions when touching the four keys
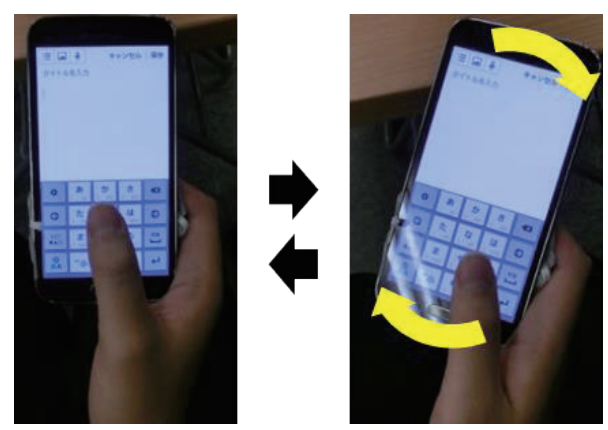

Figure 10. Rotation of a smartphone with a rubber band

\section{Conclusion}

This study investigates the effectiveness of a rubber band in improving the usability of a smartphone by measuring the IP-joint and CM-joint angles when the subject touches keys located at the corners of a Japanese keypad. By using a rubber band, the user does not have to bend the IP-joint or abduct the CM-joint because they can rotate the smartphone such that they can touch the keys easily. In future studies, the authors plan to estimate the joint angles more accurately, to investigate the relationship between joint angles and muscle load more accurately, and to repeat the experiment with more subjects.

\section{References}

Hirokawa, N. et al., "Usability of Flick Input with Fit-key on Smartphone," Proceedings of ISEM '13-Sendai (CD-ROM), Sendai 2013.

Hirokawa, N. et al., "Optimization Method of a Japanese Keypad Location for Flick Input on a Smartphone," Journal of JSEM 2015, 15 Special Issue, s125-s131, 2015.

Rohen, J. W. et al., Color Atlas of Anatomy, A photographic study of the human body, Igaku-Shoin, 388-423, 2007. (in Japanese)

Netter, F. H., Netter's Atlas of Human Anatomy, 4th Ed., Nankodo, 440-472, 2007. (in Japanese) 